\title{
Pneumocystis jirovecii and Cytomegalovirus Co-Infection in AIDS Patients
}

\author{
Joana Acar ${ }^{\mathrm{a}}$, Germana da Rocha Torres ${ }^{\mathrm{a}}$, Johanna Wagner Poti Sales ${ }^{\mathrm{b}}$, \\ Bruna Leite Marques ${ }^{\mathrm{a}}$, Renato Azambuja ${ }^{\mathrm{a}}$, Luiz Carlos Aguiar Vaz, \\ Claudia Henrique da Costa ${ }^{\mathrm{a}}$, Rogerio Rufino ${ }^{\mathrm{a}, \mathrm{c}}$
}

\begin{abstract}
A case report of a young woman with insidious dyspnea, cough and weight loss that began 3 months before hospitalization. She referred to occasional cough, wheezing, and fever during the last 2 years. Over one year before, she performed a chest CT that showed diffuse ground-glass opacity (GGO) distributed in both lungs with predominance in the upper and middle areas. She immediately received standardized treatment with sulfamethoxazole-trimethoprim (SMZTMP), and ELISA and Western Blot tests for AIDS were performed, and both were positive. Pneumocystis Jirovecci (PCJ) staining and PCR from bronchoalveolar lavage were negative. The GGO seen in CT was inconclusive. After 10 days of treatment, she progressed to being respiratory insufficient. A lung biopsy was done and revealed co-infection of cytomegalovirus (CMV) and PCJ. Although first line therapy for both PCJ and CMV was instituted early, she had an unfavorable outcome. Despite following the recommendations for diagnosis and treatment of community-acquired pneumonia in immunosuppressed patients, the patient died. The use of molecular biomethods for recognizing sulfur resistance is not usually performed in daily practice and an old question regarding the aggressiveness of co-infection of PCJ and CMV returned in this case.
\end{abstract}

Keywords: Pneumocystis pneumonia; Pneumocystosis; Acquired immunodeficiency syndrome; Cytomegalovirus; Ground-glass opacity; Oral candidiasis

\section{Introduction}

Pneumocystis pneumonia (PCP) is a common opportunistic

\footnotetext{
Manuscript accepted for publication April 25, 2013

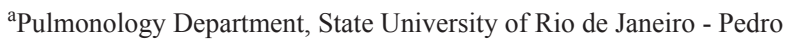
Ernesto University Hospital, Brazil

${ }^{b}$ Pathology Department, State University of Rio de Janeiro - Pedro Ernesto University Hospital, Brazil

${ }^{\mathrm{c} C o r r e s p o n d i n g ~ a u t h o r: ~ R o g e r i o ~ R u f i n o, ~ P u l m o n o l o g y ~ D e p a r t m e n t, ~}$ State University of Rio de Janeiro - Pedro Ernesto University Hospital, Brazil. Email: rrufino.uerj@gmail.com
}

doi: http://dx.doi.org/10.4021/jmc1249w infection in immunocompromised patients, especially those with acquired immunodeficiency syndrome (AIDS). Cytomegalovirus (CMV) pneumonia is a complication presented by these patients when they are in a state of severe immunosuppression. The purpose of this report is to describe a patient with PCP and CMV co-infection treated with the recommended therapy for both diseases who had an unfavorable outcome.

\section{Case Report}

A 40-year-old female, was admitted to treatment in the University Hospital in February 2012, reporting dyspnea at rest, cough, weakness and weight loss of 21 pounds in three months. She reported onset of symptoms in August 2010, with periods of dyspnea on moderate exertion, occasional wheezing, cough and high fever. She sought emergency medical care on several occasions, always being misdiagnosed as asthma exacerbated by infection. The patient presented monthly recurrence of symptoms, with progressive worsening starting in October 2011, when a chest CT was performed (Fig. 1a-d).

She reported no prior comorbidity, blood transfusion, alcohol abuse, smoking or illicit drug use. Despite having a steady partner for the last three years, she had unprotected sex. She worked as a dental assistant for the last two years and reported having cleaned contaminated material without the use of personal protective equipment.

On physical examination the patient was oriented, dehydrated, and pale with tachypnea and tachycardia. She had preserved peripheral capillary perfusion and an absence of lymphadenopathy. In the oral cavity, there were lesions suggestive of candidiasis. Blood pressure was normal and $\mathrm{O}_{2}$ saturation was $90 \%$. On auscultation, the respiratory murmur was universally audible without adventitious sounds. Examination of other systems was also unchanged.

Laboratory tests revealed normocytic and normochromic anemia. The leukocyte count was normal, but there was marked lymphopenia (238 lymphocytes) with a $\mathrm{CD}^{+}$cell count of 34 cells $/ \mathrm{mm}^{3}$. Lactate dehydrogenase was increased 2.5 times $(548 \mathrm{U} / \mathrm{L})$ the reference value. Serology was re- 


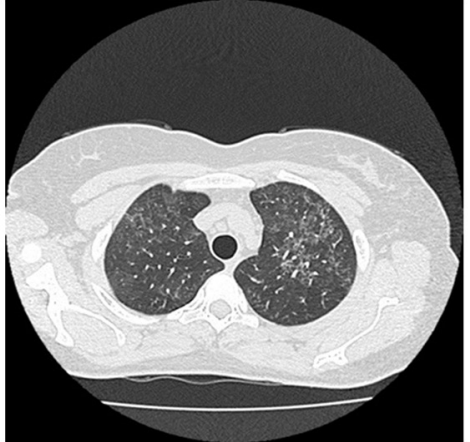

a

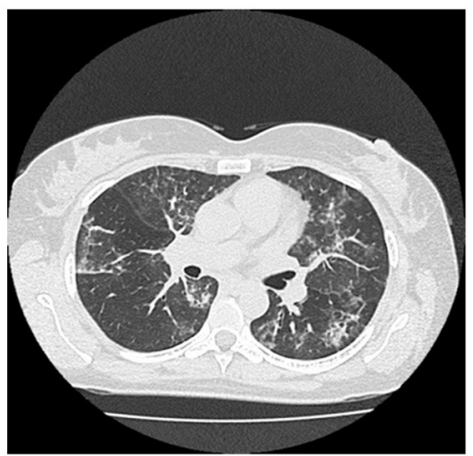

c

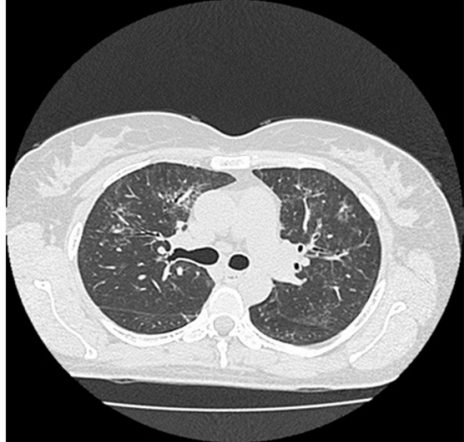

b

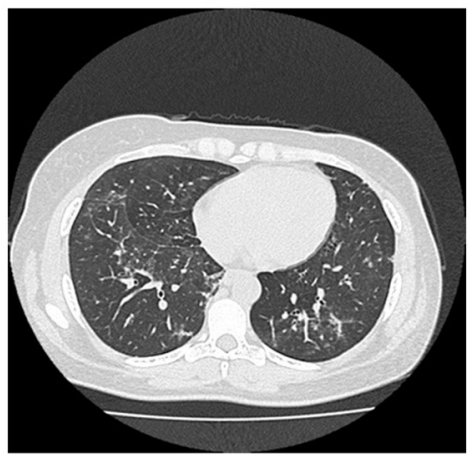

d

Figure 1. (a-d). Ground-glass opacities (GGO) distributed scarcely in the upper to lower lobes in chest-CT three months before hospitalization.

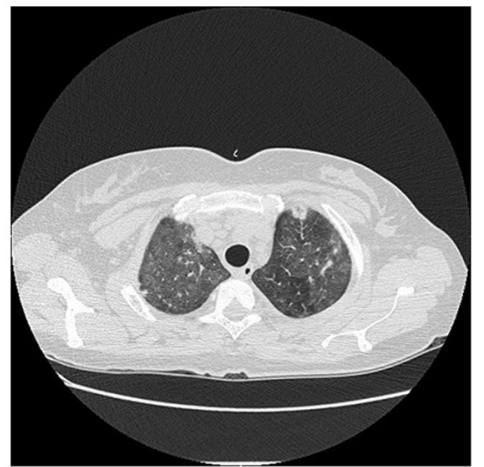

a

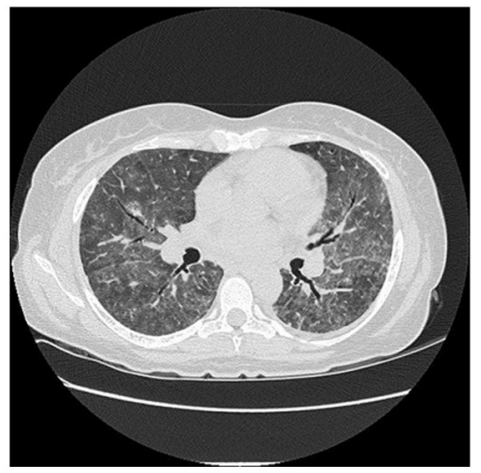

c

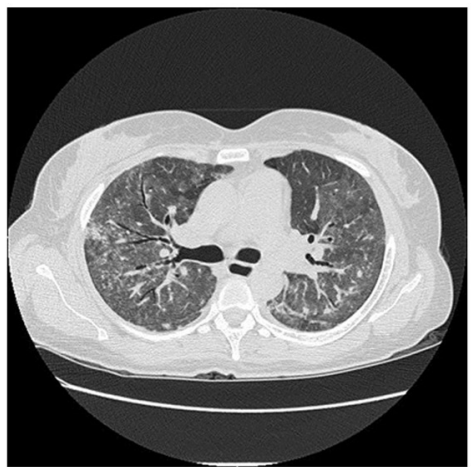

b

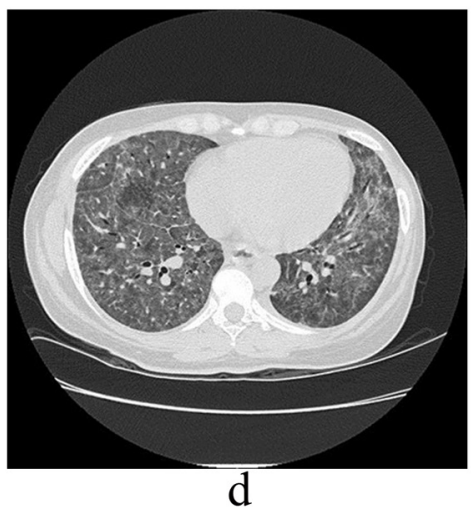

Figure 2. (a-d). During the hospitalization, GGO spread in all lobes. 


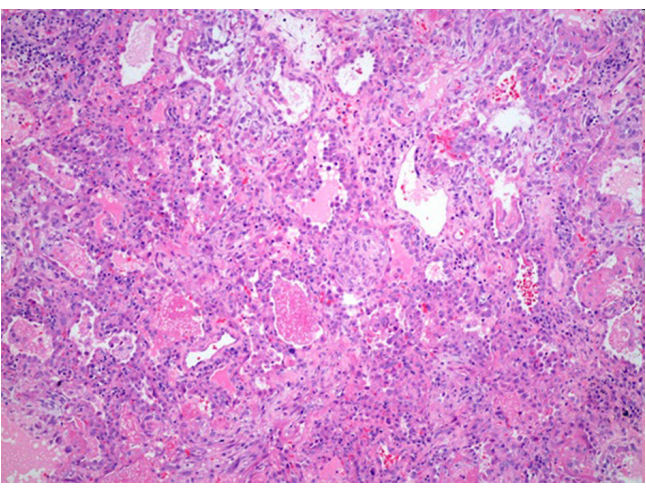

Figure 3. Lung condensation with foamy and pink exudate (P. jiroveci) filled alveoli (H\&E - $200 \times)$. The alveolar wall shows inflammation, with lymphoid infiltrates and hyaline membranes.

quested. Chest radiography revealed a bilateral diffuse interstitial infiltrate. As the principal hypothesis was PCP, empirical treatment with sulfamethoxazole/trimethropim and systemic corticosteroids was initiated, as well as oral nystatin for the candidiasis.

Bronchoscopy identified candidiasis from the oral cavity to the trachea. Bronchoalveolar lavage (BAL) of the middle lobe was performed. A direct search for fungi and acid-alcohol-resistant bacillus were negatives. Polymerase chain reaction (PCR) to test for Pneumocystis jirovecii was also negative. The HIV test was positive.

A new chest CT (Fig. 2a-d) showed increased diffuse bilateral ground glass and centrilobular nodular opacities. Peribronchial and fissure thickening were noticed, and, occasionally, micronodular pulmonary infiltrates in fissures. There was no lymphadenopathy.

Dyspnea and hypoxemia worsened and the combination of amphotericin B, ganciclovir, clarithromycin, meropenem, and vancomycin was introduced empirically. She was transferred to the intensive care unit and a lingular segmentec-

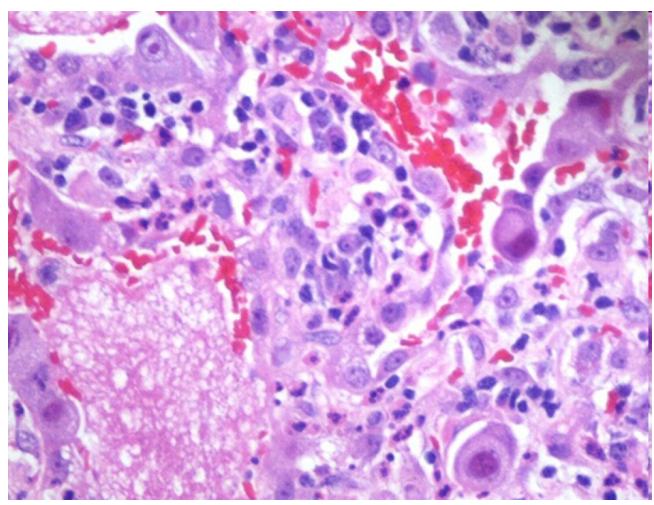

Figure 4. Violaceous to dark red intranuclear CMV inclusion with foamy exudate fills alveoli associated with Pneumocystis jirovecci $(\mathrm{H} \& \mathrm{E}-100 \times)$.

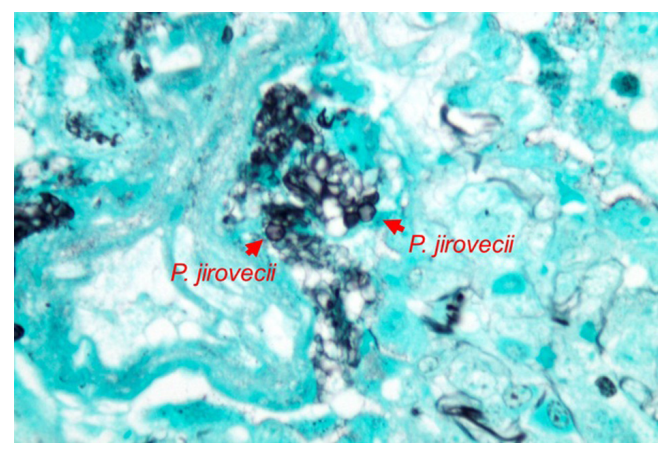

Figure 5. Clusters of Pneumocystis jirovecci (Methenamine silver stain $-100 \times$ ).

tomy was performed. After 8 days the patient died.

Histopathologic analysis of the lung biopsy revealed, macroscopically, a segment of lung measuring $5.5 \times 4.0 \mathrm{~cm}$, covered by a smooth and shiny pleura. The lung tissue was light brown and spongy. A microscopic lung fragment was seen with preserved architecture with septal thickening by marked hyperplasia of the alveolar epithelium. The alveolar space was filled with scaled pneumocystis and rosy fibrillar proteinaceous material and some hyaline membranes. There was initial fibrosis in the septum and condensation areas in the parenchyma. Immunohistochemistry for CMV showed the presence of residual virus and Grocott silver indicated the presence of Pneumocystis jirovecii (Fig. 3-6).

\section{Discussion}

Despite the introduction of antiretroviral therapy, AIDS patients with pneumopathy have many possible differential diagnoses, especially when associated with severe immunological depression. The most common causes are infections: such as PCP, bacterial pneumonia, mycobacterial, fungal, toxoplasma, Cryptococcus and Histoplasma: but lung cancer (carcinoma, lymphoma, Kaposi sarcoma), CMV, Rhodoccocus and parasitaire disease cannot be neglected $[1,2]$.

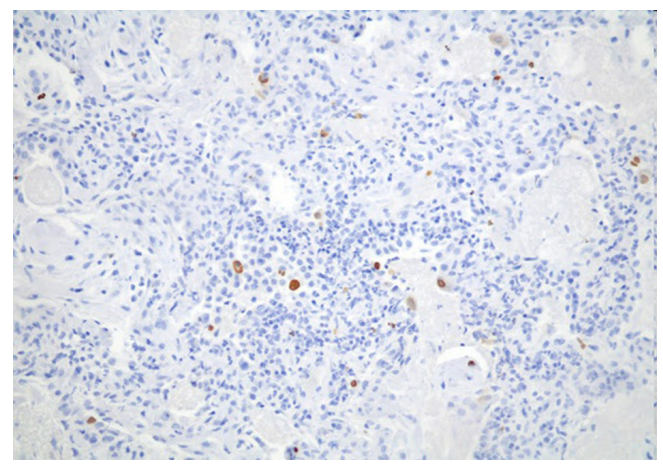

Figure 6. Strong nuclear staining for CMV may be seen. 
Pneumocystis jirovecii pneumonia is the most common cause of acute diffuse lung disease in AIDS patients with $\mathrm{CD}^{+}$counts below 200 cells $/ \mathrm{mm}^{3}$. It is characterized by dyspnea associated with hypoxemia, dry cough, increased serum LDH, and chest discomfort with or without fever. Chest X-ray typically shows bilateral interstitial infiltrate predominantly perihilar and in the lower lobes. Other forms: such as the presence of cystic lesions, cavitation, abscesses, lobar consolidation, nodular lesions, pneumothorax, pleural effusion, miliary forms, mediastinal adenopathy and even normal chest X-ray - are not common, but can occur. Thus, high-resolution chest $\mathrm{CT}$ can demonstrate disease that was not diagnosed by conventional X-ray. CT findings to be considered are: the ground-glass pattern, alveolar consolidations, bronchial dilatation, and bronchial wall and interlobular septa thickening, which may form a mosaic attenuation pattern, intralobular reticulation, cystic lesions and nodules $[1,3,4]$. Although the presence of ground-glass opacities is non-specific for PCP, their absence strongly argues against the diagnosis of PCP, and no further diagnostic testing for PCP or PCP treatment is generally warranted in these cases $[3,5]$. Bronchoscopy with BAL is considered as the gold standard procedure to diagnose PCP in HIV-infected patients and has a reported sensitivity of $98 \%$ or greater [6]. The sensitivity and specificity of PCR are high - however, the cut-off that should be used to differentiate between colonization and pneumocystis pneumonia was not established [5].

The ground-glass and alveolar opacities are due to the presence of alveolar filling with eosinophilic foamy exudate, consisting of surfactant, fibrin and cellular debris from pneumocyte injury and parasites. The nodules are due to the formation of non-caseating granulomas, the alveoli being filled by microorganisms, inflammatory cells, and necrosis. According to the inflammatory response and tissue regeneration capacity, some patients develop fibrosis. Diagnosis is by direct visualization of the fungus in tissues or fluids or PCR. Faced with a suspected case, it is important to start empirical treatment - the first choice being sulfamethoxazole-trimethoprim (SMZ-TMP) [1, 5]. The frequency of sulfamethoxazole/trimethopim resistance to treat the PCP is low. A retrospective study with 359 HIV-infected adult patients, with first-episode laboratory-confirmed Pneumocystis jirovecii pneumonia, showed that only twenty-five patients (7\%) failed co-trimoxazole treatment [7]. The overall mortality was described as $13.6 \%$, though the mortality among patients who failed co-trimoxazole treatment was 48\% [7].

The widespread use of trimethoprim-sulfamethoxazole for PCP prophylaxis has been associated with increases in trimethoprim-sulfamethoxazole-resistant bacteria and has raised concerns over potential trimethoprim-sulfamethoxazole drug resistance in $P$. jirovecii treatment [8]. The genetic mutations within the dihydrofolate reductase (DHFR) and dihydropteroate synthase (DHPS) genes, which are enzymatic targets of trimethoprim and sulfur (sulfamethoxazole and dapsone) medications, are reported in anecdotal studies $[5,8]$. These studies report a wide range in the frequency of DHPS mutations (from 3.7 to $81 \%$ ), and also reveal a geographic variation and increase in the proportion of DHPS mutations over time. Specifically, two non-synonymous mutations that result in amino acid substitutions at amino acid position 55 (Thr/Ala) and/or position 57 (Pro/Ser) are almost exclusively reported [5].

In 2005, a prospective and observational study determined the impact of Pneumocystis jirovecii DHPS gene mutations on morbidity and mortality of PCP in HIV-positive patients. The majority of patients $(86 \%)$ with PCP containing Pneumocystis DHPS mutations survived. There was a trend for more patients with mutant genotypes than patients with wild-type genotypes to require mechanical ventilation $(14.3 \%$ versus $2.5 \% ; \mathrm{P}=0.056)$ and to die $(14.3 \%$ versus $7.5 \%, \mathrm{P}=0.31$ ) [9].

CMV may cause serious disease in immunocompromised adults, especially those who have undergone organ transplantation or those with AIDS. It was estimated that CMV disease develops in $20 \%$ of HIV-infected individuals within 2 years after the $\mathrm{CD} 4^{+}$cell count falls below $100 / \mathrm{mm}^{3}$, and it can result in a wide variety of clinical presentations in AIDS, such as pneumonia, retinitis, encephalitis, hepatitis, and ulceration of the gastrointestinal tract, which are complications that are associated with high morbidity and mortality [10]. Histologic evidence of CMV infection in the lungs was described in $29 \%$ to $93 \%$ of cases [10].

Pulmonary infections caused by CMV as a sole pathogen occur only in patients in advanced stages of immunosuppression, when $\mathrm{CD}^{+}$is below 50 cells $/ \mathrm{mm}^{3}$. The most common is the co-infection of CMV with another opportunist pathogen. The diagnosis can be confirmed by serology, isolation, detection by PCR, by culture methods, or direct visualization. The first-line therapy is based on the use of inhibitors of viral DNA polymerase such as ganciclovir $[10,11]$.

The case report presented demonstrates the unfavorable clinical course of a patient with AIDS who presented a severe pneumonia by PCP and CMV co-infection, despite the use of the recommended first-line therapy of TMP-SMZ for PCP and ganciclovir for CMV. As reported, there was a suspected diagnosis and treatment was initiated after five months of the classical manifestation. It is known that when the diagnosis is delayed, PCP is associated with worse outcomes, and if not treated is invariably fatal. In a recent review of the literature, it was observed that most cases of treatment failure are due to the severity of the illness prior to diagnosis and treatment, rather than drug resistance. Simultaneous infection with other opportunistic pathogens is a potential cause of treatment failure for PCP. Thus, differential diagnoses should be employed to exclude associated lung infections. Also correlated with a worse prognosis is advanced age, previous episodes of PCP, high concentration of $\mathrm{LDH}$, low $\mathrm{CD}^{+}$count, and low level of albumin $[1,5]$. 
The degree of hypoxemia in the clinical presentation usually presents a strong correlation with the prognosis of PCP $[1,5]$. We emphasize that patients with measurement of arterial blood gases and oxygen partial pressure $\leq 70 \mathrm{mmHg}$ or alveolar-arterial gradient $\geq 35 \mathrm{mmHg}$ should receive corticosteroid therapy, as employed in this case. In patients requiring mechanical ventilation, using low tidal volumes and low-pressure plateau is recommended since the potential presence of cysts increases the risk of pneumothorax.

The specific diagnosis of PCP requires documentation of the organism in respiratory samples. BAL is an option, with or without transbronchial biopsy. BAL alone has good performance in patients infected with HIV. Some studies have demonstrated that combining immunofluorescence with BAL increases sensitivity to $80-98 \%$ [12]. The use of PCR in addition to the diagnosis may allow quantification of the organism in an attempt to differentiate between infection and colonization, and could be used to detect drug resistance. Studies show that the use of PCR in diagnosis of PCP from BAL samples has sensitivities ranging from $81-100 \%$ and specificities $86-100 \%[12,13]$. Transthoracic needle biopsy has a high diagnostic yield, but is rarely used in these cases due to the incidence of pneumothorax. Lung biopsy by thoracotomy or video-assisted surgery can be performed with nearly $100 \%$ sensitivity and specificity for the diagnosis of PCP, which defined the diagnosis of our patient.

\section{Conclusion}

The co-infection of Pneumocystis jirovecii with other opportunistic pathogens, such as cytomegalovirus, should be part of the routine investigation of lung disease in immunocompromised patients who do not obtain satisfactory clinical response within four days of treatment with SMZ-TMP. Proper diagnosis and early introduction of therapy are essential to the approach. PCP should be considered with CMV as a co-infection in patients with severe hypoxemia and low $\mathrm{CD} 4^{+}$lymphocyte count, and one should take into account that they might have an unfavorable prognosis.

\section{Conflict of Interest}

None.

\section{References}

1. Thomas CF, Jr., Limper AH. Pneumocystis pneumonia.
N Engl J Med. 2004;350(24):2487-2498.

2. Sepkowitz KA. Opportunistic infections in patients with and patients without Acquired Immunodeficiency Syndrome. Clin Infect Dis. 2002;34(8):1098-1107.

3. De Castro N, Scemla A, Gallien S, Molina JM. [Pneumocystis jirovecii pneumonia in HIV-infected patients]. Rev Mal Respir. 2012;29(6):793-802.

4. Kanne JP, Yandow DR, Meyer CA. Pneumocystis jiroveci pneumonia: high-resolution CT findings in patients with and without HIV infection. AJR Am J Roentgenol. 2012;198(6):W555-561.

5. Huang L, Cattamanchi A, Davis JL, den Boon S, Kovacs J, Meshnick S, Miller RF, et al. HIV-associated Pneumocystis pneumonia. Proc Am Thorac Soc. 2011;8(3):294-300.

6. Huang L, Hecht FM, Stansell JD, Montanti R, Hadley WK, Hopewell PC. Suspected Pneumocystis carinii pneumonia with a negative induced sputum examination. Is early bronchoscopy useful? Am J Respir Crit Care Med. 1995;151(6):1866-1871.

7. Fisk M, Sage EK, Edwards SG, Cartledge JD, Miller RF. Outcome from treatment of Pneumocystis jirovecii pneumonia with co-trimoxazole. Int J STD AIDS. 2009;20(9):652-653.

8. Huang L, Crothers K, Atzori C, Benfield T, Miller R, Rabodonirina M, Helweg-Larsen J. Dihydropteroate synthase gene mutations in Pneumocystis and sulfa resistance. Emerg Infect Dis. 2004;10(10):1721-1728.

9. Crothers K, Beard CB, Turner J, Groner G, Fox M, Morris A, Eiser S, et al. Severity and outcome of HIV-associated Pneumocystis pneumonia containing Pneumocystis jirovecii dihydropteroate synthase gene mutations. AIDS. 2005;19(8):801-805.

10. Salomon N, Perlman DC. Cytomegalovirus pneumonia. Semin Respir Infect. 1999;14(4):353-358.

11. Whitley RJ, Jacobson MA, Friedberg DN, Holland GN, Jabs DA, Dieterich DT, Hardy WD, et al. Guidelines for the treatment of cytomegalovirus diseases in patients with AIDS in the era of potent antiretroviral therapy: recommendations of an international panel. International AIDS Society-USA. Arch Intern Med. 1998;158(9):957969.

12. Khan MA, Farrag N, Butcher P. Diagnosis of Pneumocystis carinii pneumonia: immunofluorescence staining, simple PCR or nPCR. J Infect. 1999;39(1):77-80.

13. Oren I, Hardak E, Finkelstein R, Yigla M, Sprecher H. Polymerase chain reaction-based detection of Pneumocystis jirovecii in bronchoalveolar lavage fluid for the diagnosis of pneumocystis pneumonia. Am J Med Sci. 2011;342(3):182-185. 